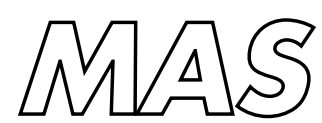

Modelling, Analysis and Simulation

Modelling, Analysis and Simulation
MAS Adaptive manifold-mapping using multiquadric
interpolation applied to linear actuator design 
Centrum voor Wiskunde en Informatica (CWI) is the national research institute for Mathematics and Computer Science. It is sponsored by the Netherlands Organisation for Scientific Research (NWO).

CWI is a founding member of ERCIM, the European Research Consortium for Informatics and Mathematics.

CWI's research has a theme-oriented structure and is grouped into four clusters. Listed below are the names of the clusters and in parentheses their acronyms.

Probability, Networks and Algorithms (PNA)

Software Engineering (SEN)

\section{Modelling, Analysis and Simulation (MAS)}

Information Systems (INS)

Copyright (C) 2006, Stichting Centrum voor Wiskunde en Informatica

P.O. Box 94079, 1090 GB Amsterdam (NL)

Kruislaan 413, 1098 SJ Amsterdam (NL)

Telephone +31205929333

Telefax +31205924199

ISSN 1386-3703 


\title{
Adaptive manifold-mapping using multiquadric interpolation applied to linear actuator design
}

\begin{abstract}
In this work a multilevel optimization strategy based on manifold-mapping combined with multiquadric interpolation for the coarse model construction is presented. In the proposed approach the coarse model is obtained by interpolating the fine model using multiquadrics in a small number of points. As the algorithms iterates, the response surface model is improved by enriching the set of interpolation points. This approach allows to accurately solve the TEAM Workshop Problem 25 using as little as 33 finite element simulations. Furthermore is allows a robust sizing optimization of a cylindrical voice-coil actuator with seven design variables. Further analysis is required to gain a better understand of the role that the initial coarse model accuracy plays the convergence of the algorithm. The proposed allows to carry out such analysis by varying the number of points included in the initial response surface model. The effect of the trust-region stabilization in the presence of manifolds of equivalent solutions is also a topic of further investigations.
\end{abstract}

2000 Mathematics Subject Classification: 65K10, 65M60, 65N55, 65Y20, 90C31

Keywords and Phrases: Multiquadric interpolation; Manifold-mapping; Actuators

Note: This research was supported by the Dutch Ministry of Economic Affairs within the project IOP-EMVT 04302. 



\title{
Adaptive Manifold-Mapping Using Multiquadric Interpolation Applied to Linear Actuator Design
}

\author{
D. Lahaye ${ }^{1 *}$, A. Canova ${ }^{2 \dagger}$, G. Gruosso ${ }^{3 \ddagger}$ and M. Repetto ${ }^{2 \S}$ \\ ${ }^{1}$ Centrum voor Wiskunde en Informatica (CWI) \\ Kruislaan 413, 1090 GB, Amsterdam, The Netherlands \\ 2 Dipartimento di Ingegneria Elettrica, Politecnico di Torino \\ Corso Duca degli Abruzzi, 29, I-10129 Italy \\ ${ }^{3}$ Dipartimento di Elettronica e Informazione, Politecnico di Milano \\ Piazza Leonardo da Vinci, 32, Milano, I-20133, Italy
}

\begin{abstract}
Purpose - In this work a multilevel optimization strategy based on manifold-mapping combined with multiquadric interpolation for the coarse model construction is presented.

Design/methodology/approach - In the proposed approach the coarse model is obtained by interpolating the fine model using multiquadrics in a small number of points. As the algorithms iterates, the response surface model is improved by enriching the set of interpolation points.

Findings - This approach allows to accurately solve the TEAM Workshop Problem 25 using as little as 33 finite element simulations. Furthermore is allows a robust sizing optimization of a cylindrical voice-coil actuator with seven design variables.

Research limitations/implications - Further analysis is required to gain a better understand of the role that the initial coarse model accuracy plays the convergence of the algorithm. The proposed allows to carry out such analysis by varying the number of points included in the initial response surface model. The effect of the trust-region stabilization in the presence of manifolds of equivalent solutions is also a topic of further investigations.

Originality/value - Unlike the closely related space-mapping algorithm, the manifold-mapping algorithm is guaranteed to converge to an fine model optimal solution. By combining it with multiquadric response surface models, we extend its applicability to problems for which other kinds of coarse model such as lumped parameter approximations for instance are tedious or impossible to construct.
\end{abstract}

Keywords Multiquadric interpolation, Manifold-mapping, Actuators Paper Type Research paper

\footnotetext{
*Phone: +31 20592 4122, E-mail: d.lahaye@cwi.nl

†Phone: +390115647 158, E-mail: aldo.canova@polito.it

‡Phone: +39022 3993696 E-mail:gruosso@elet.polimi.it

$\S$ E-mail: maurizio.repetto@polito.it
} 


\section{Introduction}

The space-mapping technique [1] allows to speed up computationally expensive simulation-based optimization procedures through the use of approximate models. In the space-mapping literature the so-called fine and coarse models are conceived as mappings from the design space to the space of model responses. The key element is the space-mapping function. It reparametrises the coarse model domain in such a way to minimize the discrepancy between the fine and coarse model responses. The composition of the space-mapping function and the coarse model defines a surrogate for the fine model. Instead of solving the fine model optimization problem directly, space-mapping solves the surrogate optimization problem through a sequence of approximations of the space-mapping function. In turn this sequence defines a sequence of coarse model optimization problems whose solutions converge by definition to the space-mapping solution. The computational efficiency of this approach stems from the fact that it takes less fine model evaluations for the sequences mentioned afore to converge than it takes to solve the fine model optimization problem. The drawback is that the the space-mapping solution does not necessarily coincide with fine model optimum.

In the manifold-mapping technique [5], the surrogate model is constructed in such a way that in a neighbourhood of the fine model optimum, the surrogate model response closely resembles its fine model counterpart. This guarantees that the solutions of the surrogate and fine model optimization problem do coincide. The space-mapping function is replaced by the so-called manifold-mapping function. The latter is an affine transformation between tangent manifolds of the coarse and fine model image spaces. Manifold-mapping is computationally as efficient as space-mapping.

Optimization problems encountered in engineering practise are often illposed and this adversely affects the convergence of optimization procedures. To overcome this problem, some kind of stabilization is required. In this work we will make use of a trust-region strategy [8] that prevents the difference in the successive manifold-mapping iterands from becoming too large.

The efficiency of manifold-mapping critically hinges on the the balance between quality and computational cost of the coarse model. In this paper we advocate the use of response surface coarse models. A fairly common two-stage approach in applying such models is the following. An initial response surface model is build and optimized using a global optimizer. In a second stage points in regions of the design space in which a minimum is found is added to the response surface model. In this approach the choice of the points to add to the response surface is entirely based upon heuristics. In combining manifoldmapping with a response surface methodology we distinguish the following four advantages. Firstly, it alleviates the burden of having to construct accurate response surface models. Secondly, the response surface can be adaptively improved during the manifold-mapping iterative process by enriching the set of interpolation points with points for which manifold-mapping computes the fine model response. This iterative model refinement requires negligible computational cost. Thirdly, the number and location of the initial interpolation points can in principle be varied to investigate what kind of coarse model accuracy is required for a particular application. And finally, the use of response surface models offers a gateway to applied manifold-mapping optimization to problems 
for which other kinds of coarse model such as lumped parameter approximations for instance are tedious or impossible to construct.

This paper is organized as follows: In Section 2 we introduce the spacemapping technique. In Section 3 we give the details of the response surface construction using multiquadrics. In Section 4 we explain the manifold-mapping algorithm and its combined use with response surface coarse models. In Section 5 we give evidence of the performance of the proposed strategy by means of two design problems: the TEAM Workshop Problem 25 and the sizing optimization of a cylindrical voice-coil actuator.

\section{The Space-Mapping Technique}

Let us consider an optimization problem with design variables $\mathbf{x}$ in the design space $\mathbf{x} \in X \subset \mathbb{R}^{n}$ and specifications $\mathbf{y} \in \mathbb{R}^{m}$. The accurate behavior of electromechanical devices is often studied using models that have large computational costs, e.g., finite element models. In space-mapping terminology [1] these models are called fine models. The fine model response is denoted by $\mathbf{f}(\mathbf{x}) \in \mathbb{R}^{m}$. The problem we set out to solve in this paper can be stated as

$$
\text { find } \mathbf{x}_{f}^{*} \in X \text { such that } \mathbf{x}_{f}^{*}=\underset{\mathbf{z} \in X}{\operatorname{argmin}}\|\mathbf{f}(\mathbf{z})-\mathbf{y}\|,
$$

where argmin denotes the argument of the minimum. Space-mapping needs a second, possibly less accurate but computationally cheaper model, called coarse model. The coarse models are assumed to be defined over the same design space $X$. Their response is denoted by $\mathbf{c}(\mathbf{x}) \in \mathbb{R}^{m}$. The auxiliary optimization problem can be formulated as

$$
\text { find } \mathbf{x}_{c}^{*} \in X \text { such that } \mathbf{x}_{c}^{*}=\underset{\mathbf{z} \in X}{\operatorname{argmin}}\|\mathbf{c}(\mathbf{z})-\mathbf{y}\| .
$$

The space-mapping function $\mathbf{p}: X \mapsto X$ maps the design variable $\mathbf{x}$ with corresponding fine model response $\mathbf{f}(\mathbf{x})$ to the design variable $\mathbf{p}(\mathbf{x})$ that minimizes the fine and coarse model discrepancy, i.e.

$$
\mathbf{x} \mapsto \mathbf{p}(\mathbf{x})=\underset{\mathbf{z} \in X}{\operatorname{argmin}}\|\mathbf{c}(\mathbf{z})-\mathbf{f}(\mathbf{x})\|,
$$

This function allows to defined the surrogate $\mathbf{c}(\mathbf{p}(\mathbf{x}))$ for $\mathbf{f}(\mathbf{x})$. The spacemapping solution is defined through the use of this surrogate as follows

$$
\text { find } \mathbf{x}_{s m}^{*} \in X \text { such that } \mathbf{x}_{s m}^{*}=\underset{\mathbf{z} \in X}{\operatorname{argmin}}\|\mathbf{c}(\mathbf{p}(\mathbf{z}))-\mathbf{y}\| .
$$

In practise (4) is solved iteratively by replacing $\mathbf{p}(\mathbf{x})$ by an sequence of approximation $\left\{\mathbf{p}_{k}(\mathbf{x})\right\}_{k \geq 1}$ converging to $\mathbf{p}(\mathbf{x})$ for $k \rightarrow \infty$ yielding a sequence of iterands $\left\{\mathbf{x}_{k, s m}\right\}_{k \geq 1}$ converging to $\mathbf{x}_{s m}^{*}$. Typically $\mathbf{p}(\mathbf{x})$ is expanded in a first order Taylor series and Broyden's method is employed to update the gradient $\nabla \mathbf{p}(\mathbf{x})$ from iteration $k$ to $k+1$. The space-mapping technique thus replaces the fine model optimization problem (1) by the surrogate problem (4) and solves the latter by the following sequence of coarse model optimization problems

$$
\text { find } \mathbf{x}_{k, s m} \in X \text { such that } \mathbf{x}_{k, s m}=\underset{\mathbf{z} \in X}{\operatorname{argmin}}\left\|\mathbf{c}\left(\mathbf{p}_{k}(\mathbf{z})\right)-\mathbf{y}\right\|,
$$


each requiring a fine model evaluation to compute $\mathbf{p}_{k}(\mathbf{x})$. However, we have that

$$
\mathbf{x}_{s m}^{*} \neq \mathbf{x}_{f}^{*}
$$

unless additional assumptions on $\mathbf{p}(\mathbf{x})$ (and thus indirectly on the coarse model) are imposed.

\section{Response Surface Modelling}

Response surface models have been known for a long time and a well-established theory exists $[2,10]$. Originally developed for optimization based on real experiments, they were extended to simulation-based optimization later on. These models are build by interpolating the fine model in a discrete set of sampling points. The two main aspects of response surface modelling are the choice of interpolating functions and of the sample points $[4,9,3]$.

In this work the response surface coarse model is constructed by interpolating the fine model $\mathbf{f}(\mathbf{x})$ in a uniform $N$-point sampling of the design space $X$ using multiquadrics. Assume first that $m=1$. The multiquadric $q_{j}(\mathbf{x}), j=1, \ldots, N$, centered on the point $\mathbf{x}_{j} \in X$ is defined as

$$
q_{j}(\mathbf{x})=\sqrt{\left\|\mathbf{x}-\mathbf{x}_{j}\right\|^{2}+h},
$$

where the parameter $h$ (called the shift-parameter) allows to control the curvature of $q_{j}(\mathbf{x})$. It is chosen by the relationship $h=\left(\sum_{j=1}^{N}\left\|\mathbf{x}_{j}\right\|\right) /(K N)$, where $K$ is an arbitrary constant, here set equal to 50 in accordance with [3]. The interpolating coarse model $\mathbf{c}(\mathbf{x})$ can then be expressed as

$$
\mathbf{c}(\mathbf{x})=\sum_{j=1}^{N} \alpha_{j} q_{j}(\mathbf{x}) .
$$

The coefficients $\alpha_{j}$ are the solution of the linear system of equations

$$
\mathbf{Q} \boldsymbol{\alpha}=\mathbf{F},
$$

where $\mathbf{Q}$ is the matrix of interpolation functions evaluated in the interpolation points $\mathbf{Q}_{i j}=q_{j}\left(\mathbf{x}_{i}\right), \boldsymbol{\alpha}=\left(\alpha_{1}, \alpha_{2}, \ldots, \alpha_{N}\right)$ the vector of coefficients and $\mathbf{F}=\left(\mathbf{f}\left(\mathbf{x}_{1}\right), \mathbf{f}\left(\mathbf{x}_{2}\right), \ldots, \mathbf{f}\left(\mathbf{x}_{N}\right)\right)$ the vector of the fine model evaluated in the interpolation points respectively. The extension of this discussion to $m>1$ is straightforward. For a giving sampling of $X$, the linear system (9) can be solved prior to the coarse model optimization iterative procedure.

The versatility of multiquadratic interpolation in representing complex isosurfaces on the cost-function was shown in e.g. [3].

\section{Adaptive Manifold-Mapping}

\subsection{The Manifold-Mapping Technique}

The manifold-mapping technique [5] exploits coarse model information and defines a surrogate optimization problem whose solution, unlike in (6), does coincide with $x_{f}^{*}$. The key ingredient is the manifold-mapping function between the 
coarse and fine model image spaces $\mathbf{c}(X) \subset \mathbb{R}^{m}$ and $\mathbf{f}(X) \subset \mathbb{R}^{m}$. This function $\mathbf{S}: \mathbf{c}(X) \mapsto \mathbf{f}(X)$ maps the point $\mathbf{c}\left(\mathbf{x}_{f}^{*}\right)$ to $\mathbf{f}\left(\mathbf{x}_{f}^{*}\right)$ and the tangent space of $\mathbf{c}(X)$ at $\mathbf{x}_{f}^{*}$ to the tangent space of $\mathbf{f}(X)$ at $\mathbf{x}_{f}^{*}$. It allows to define the surrogate model $\mathbf{S}(\mathbf{c}(\mathbf{x}))$ and the manifold-mapping solution as follows

$$
\text { find } \mathbf{x}_{m m}^{*} \in X \text { such that } \mathbf{x}_{m m}^{*}=\underset{\mathbf{z} \in X}{\operatorname{argmin}}\|\mathbf{S}(\mathbf{c}(\mathbf{z}))-\mathbf{y}\| \text {. }
$$

The manifold-mapping function $\mathbf{S}(\mathbf{x})$ is approximated by a sequence $\left\{\mathbf{S}_{k}(\mathbf{x})\right\}_{k \geq 1}$ yielding a sequence of iterands $\left\{\mathbf{x}_{k, m m}\right\}_{k \geq 1}$ converging to $\mathbf{x}_{m m}^{*}$. The individual iterands are defined by coarse model optimization

$$
\text { find } \mathbf{x}_{k, m m}^{*} \in X \text { such that } \mathbf{x}_{k, m m}=\underset{\mathbf{z} \in X}{\operatorname{argmin}}\left\|\mathbf{S}_{k}(\mathbf{c}(\mathbf{z}))-\mathbf{y}\right\| \text {. }
$$

At each iteration $k$, the construction of $\mathbf{S}_{k}$ requires the singular value decomposition of the matrices $\triangle C_{k}$ and $\triangle F_{k}$ of size $m \times \min (k, n)$ whose columns span the coarse and fine model tangent space in the current iterand, respectively. Denoting these singular value decompositions by

$$
\triangle C_{k}=U_{k, c} \Sigma_{k, c} V_{k, c}^{T} \text { and } \triangle F_{k}=U_{k, f} \Sigma_{k, f} V_{k, f}^{T},
$$

we introduce the updated objective $\mathbf{y}_{k}$ as

$$
\mathbf{y}_{k}=\mathbf{c}\left(\mathbf{x}_{k}\right)-\left[\triangle C_{k} \triangle F_{k}^{\dagger}+\left(I-U_{k, c} U_{k, c}^{T}\right)\right]\left(\mathbf{f}\left(\mathbf{x}_{k}\right)-\mathbf{y}\right)
$$

where superscript ${ }^{\dagger}$ denotes the pseudo-inverse. With this notation, the problem (11) can shown be to be asymptotically equivalent to

$$
\text { find } \mathbf{x}_{k, m m}^{*} \in X \text { such that } \mathbf{x}_{k, m m}=\underset{\mathbf{z} \in X}{\operatorname{argmin}} \|\left(\mathbf{c}(\mathbf{z})-\mathbf{y}_{k} \|\right.
$$

By construction

$$
\mathbf{x}_{m m}^{*}=x_{f}^{*} .
$$

In [6] it was shown how an inaccurate course model delivers a speedup with a factor between 4 and 5 for a design problem for a cylindrical voice-coil actuator with two design variables. Due to the ill-posedness of the problem, it is necessary to incorporate a regularization strategy to obtain similar results in higher dimensions. Such a regularization strategy is discussed in the next subsection.

\subsection{Trust-Region Strategy for Manifold-Mapping}

A bad conditioning of the matrix $\triangle F_{k}$ in (13) may result in an arbitrary large step size $\mathbf{x}_{k}-\mathbf{x}_{k-1}$. To prevent manifold-mapping from taking such steps, a trust-region stabilization was devised in [8]. In this approach, a LevenbergMarquardt-type regularization simultaneously counteracts the undesired effect of small $\triangle F_{k}$ singular values. Iterations with a too large increase of the costfunction are rejected. The use of this trust-region approach typically requires more than one fine model evaluation per manifold-mapping iteration. 


\subsection{Adaptive Improvement of the Coarse Model}

During the manifold-mapping iteration, the points $\mathbf{x}_{k}$ for with the algorithm evaluates $\mathbf{f}\left(\mathbf{x}_{k}\right)$ can be added to set of response surface interpolation points. By doing so, the response surface is adaptively improved. Each time the set of interpolation points is enriched, the linear system (9) has to be solved for the coefficients $\boldsymbol{\alpha}$. This can be done prior to entering the coarse model iterative optimization procedure and at computational cost much smaller than required for a fine model evaluation.

\section{$5 \quad$ Numerical Results}

In this section the performance of the proposed optimization strategy is demonstrated on two problems: the well-documented TEAM Workshop Problem 25 $[13,12]$ and the first of two cylindrical voice-coil actuator design problems introduced in $[6,7]$.

The goal of the TEAM problem 25 is to optimize the shape of ferromagnetic die molds in such a way obtain a magnetic flux in the cavity as radial and as close to $.35 \mathrm{~T}$ as possible. The design space $X \subset \mathbb{R}^{4}$ is a hypercube defined by bound constraints on the design variables. The fine model consists of a finite element discretization of the perpendicular current vector potential formulation, using isoparametric second order Lagrangian elements on unstructured meshes of triangles. Two adaptive refinement steps result in a discrete problem with between 10000 and 12500 triangles and between 20000 and 25000 degrees of freedom, depending on the design parameters. The fine model response $\mathbf{f}(\mathbf{x}) \in \mathbb{R}^{20}$ and specifications $\mathbf{y} \in \mathbb{R}^{20}$ represent the computed and the specified magnetic flux components $B_{x}$ and $B_{y}$ computed at 10 points in the cavity respectively.

The goal of the voice-coil actuator problem is to find the size of different components of the actuator, in such a way as to obtain a magnetic force on the coil over the $5 \mathrm{~mm}$ stroke as constant and as close to $24 \mathrm{~N}$ as possible. The space $X \subset \mathbb{R}^{7}$ is defined by bound and additional linear inequality constraints that ensure that the coil positions remain feasible. The fine model is a finite element discretization of the azimuthal current vector potential formulation. A single adaptive refinement step results in a problem with between 5000 and 10000 triangles and between 11000 and 18000 degrees of freedom depending on the design variables and the position of the coil. The fine model response $\mathbf{f}(\mathbf{x}) \in \mathbb{R}^{9}$ gives the computed magnetic force on the coil in 9 coil positions.

In both problems, the initial response surface coarse model is constructed by an uniform sampling of $X$. For each design variables, the interval from lower to upper bound is subdivided into an equidistant grid of four intervals. As interpolation points the Cartesian product of the first or third interior node on each axis is chosen, resulting e.g. in problems with four design variables in 16 interpolation points in total. This coarse model will be referred to as the $2 \times 2 \times 2 \times 2$ coarse model later on. Analogously, the coarse model with the second interior point along each axis added to the set of interpolation points will be abbreviated as the $3 \times 3 \times 3 \times 3$ coarse model. In the voice-coil actuator problem the response surface is constructed for 9 positions of the coil.

The bulk of the coarse model computational cost involves solving the linear system (9) for $\boldsymbol{\alpha}$ and is small compared with a finite element analysis. This 
allows a stochastic algorithm to be used for a coarse model optimization (11). For this purpose we use differential evolution [11]. We set the parameters in our code used such that at each manifold-mapping iteration, the coarse model optimizer requires a total of 1250 and 5000 function evaluations in the four and seven dimensional problems, respectively.

In our numerical test we give evidence of both the stability and efficiency of our approach and we point out which algorithmic components are responsible for this efficiency. As our algorithm contains a stochastic component, we've chosen to report on 50 consecutive runs of the algorithm under the same conditions and to compare the minimal cost-function values and the number of fine-model evaluations of these runs.

\subsection{TEAM Workshop Problem 25: Die Mold Press}

In the TEAM 25 problem, we run 50 times 20 iterations of two variants of manifold-mapping without trust-region stabilization. Without this stabilization, each manifold-mapping iteration requires only one fine model evaluation. As however the convergence of the algorithm is in general non-monotone, the minimal cost-function value in a single run of 20 iterations can be found in less than 20 fine model evaluations. Taking into account that the cost function (cfr. [12]) carries the unit of the square of the magnetic flux $\left(\mathrm{T}^{2}\right)$, our results can be summarized as follows. Without adaptive coarse model refinement, the algorithm finds on average a minimal cost-function value $\left\|\mathbf{f}\left(\mathbf{x}_{m m}^{*}\right)-\mathbf{y}\right\|=5.5 e-3 \mathrm{~T}^{2}$ with a standard deviation of $2.1 e-3 \mathrm{~T}^{2}$. The adaptive refinement has a enormous impact on both quality and the variation of the solution found. On average the minimal cost-function drops by a factor of 20 to $\left\|\mathbf{f}\left(\mathbf{x}_{m m}^{*}\right)-\mathbf{y}\right\|=2.7 e-4 \mathrm{~T}^{2}$ and the standard deviation by a factor of 25 to $8.6 e-5 \mathrm{~T}^{2}$. Once the initial coarse model has been constructed, the adaptive manifold-mapping algorithm finds such high quality solutions using on the average only seventeen fine model evaluations! In total the algorithm requires on the average 33 finite element simulations to solve the problem.

A detail of the optimal geometry and flux distribution are shown in Figure 1.

\subsection{Cylindrical Voice Coil Actuator}

To solve the voice-coil actuator problem, we adopt a progressive approach on the number of design variables. By this we mean that we initially seek an optimal solution by varying the first and the first two design variables only, keeping all other values fixed at their initial value. In a second and third stage we gradually increase the complexity of the problem by letting the first four and all seven variables to come into play, respectively. This approach offers the straightforward stability test on the algorithm of verifying that the cost function decreases as expected as more degrees of freedom are introduced. In the following summarise the numerical results for the three afore mentioned stages. As fine model we consider a finite element model with a linear ferromagnetic core. 


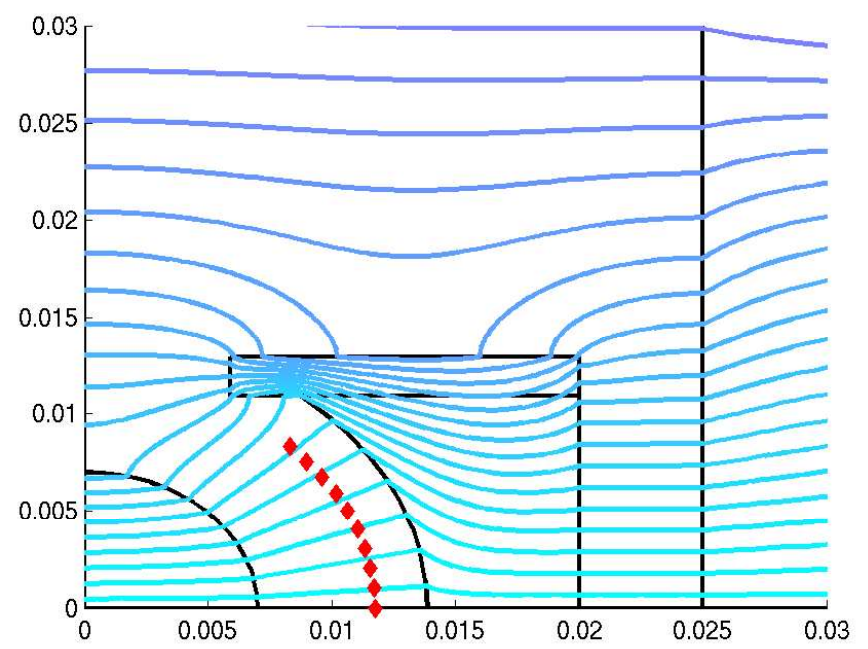

(a) Optimal geometry

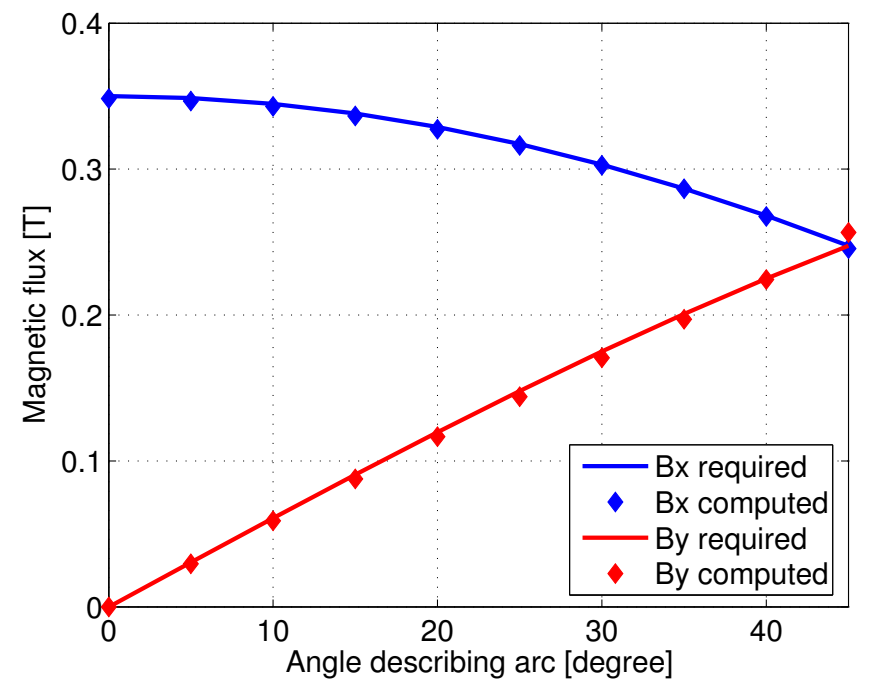

(b) Optimal flux distribution

Figure 1: Detail of the optimal geometry and flux distribution for the TEAM 25 problem. 


\subsubsection{Results for one and two design variables}

In [6] the following two results were shown. Using $x_{1}$ as single design variable, the minimum cost-function value equals $\left\|\mathbf{f}\left(\mathbf{x}_{m m}^{*}\right)-\mathbf{y}\right\|=6.5 e-2$. Using $x_{1}$ and $x_{2}$ as design variables, the cost-function contour plot shows that the $1 \mathrm{D}$ optimum lies in a long and steep valley of points with approximately the same cost-function value.

\subsubsection{Results for four design variables}

For the problem with four design variables, we run 50 times 40 iterations of manifold-mapping with adaptive coarse model refinement and without trustregion on an $2 \times 2 \times 2 \times 2$ initial coarse model. On average the minimal costfunction value found is equal to $\left\|\mathbf{f}\left(\mathbf{x}_{m m}^{*}\right)-\mathbf{y}\right\|=1.6 e-2$, with a standard deviation of 3.7e-4. Having build the initial coarse model, adaptive manifold mapping finds these solutions using on average 37 finite element simulations. The fact that the $4 \mathrm{D}$-optimum is significantly better than its $2 \mathrm{D}$ counterpart provides an indication for the stability of the algorithm.

\subsubsection{Results for seven design variables}

Numerical tests indicated that an initial coarse model with 2 points along each axis was not sufficiently accurate to solve the seven-dimensional problem. We therefore resorted to both a finer initial coarse model and a trust-region stabilization of the algorithm. We run 50 times 50 iterations of manifold-mapping with adaptive coarse model refinement on an $2 \times 2 \times 2 \times 2 \times 3 \times 3 \times 3$ coarse model. The average cost function reduces by more than a factor two compared with the four-dimensional results and is equal to $\left\|\mathbf{f}\left(\mathbf{x}_{m m}^{*}\right)-\mathbf{y}\right\|=6.3 e-3$ with a standard deviation of $2.1 e-3$. The trust-region stabilization makes the algorithm more expensive per iteration and in this experiment an average of 166 finite element simulation per run are required.

A seven-dimensional optimal geometry and the force-displacements curves corresponding to 2D, 4D and 7D optimal configurations are given in Figure 2.

\section{Conclusions}

In this work the ability of manifold-mapping of exploiting coarse model information to speed up the convergence of expensive optimization procedures was combined with versatility of multiquadratic interpolation of representing the cost-function isosurfaces. We showed the efficiency of the adaptive manifoldmapping algorithm in solving the TEAM Workshop Problem 25 using as little as 33 finite element simulations and its the robustness in the progressive sizing optimization of a cylindrical voice-coil actuator. The strategy proposed opens perspectives to solve more challenging electromagnetic optimization problems.

\section{Acknowledgement}

The first author would like to thank David Echeverría, Herbert De Gersem and Pieter Hemker for their valuable input during the course of this research. The 


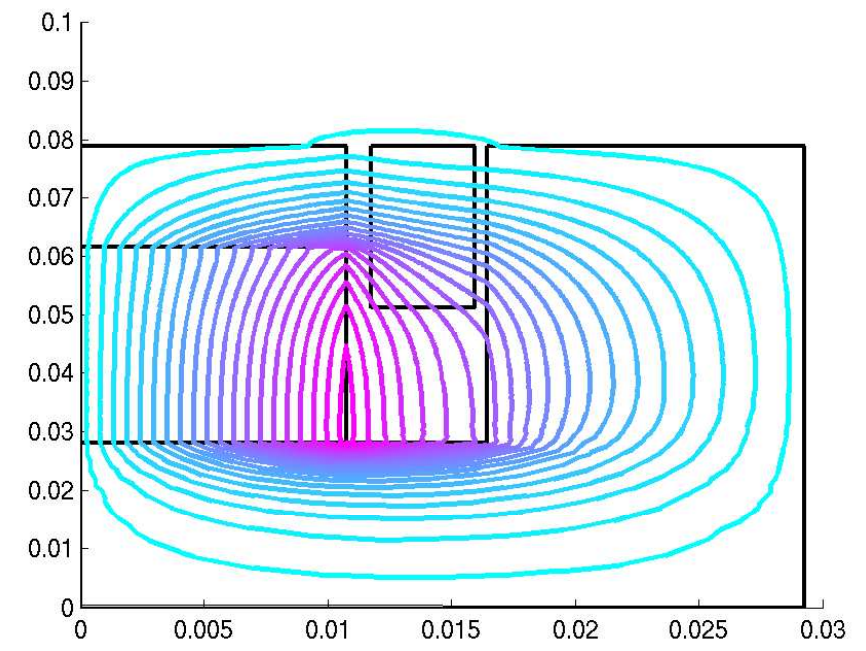

(a) Optimal geometry

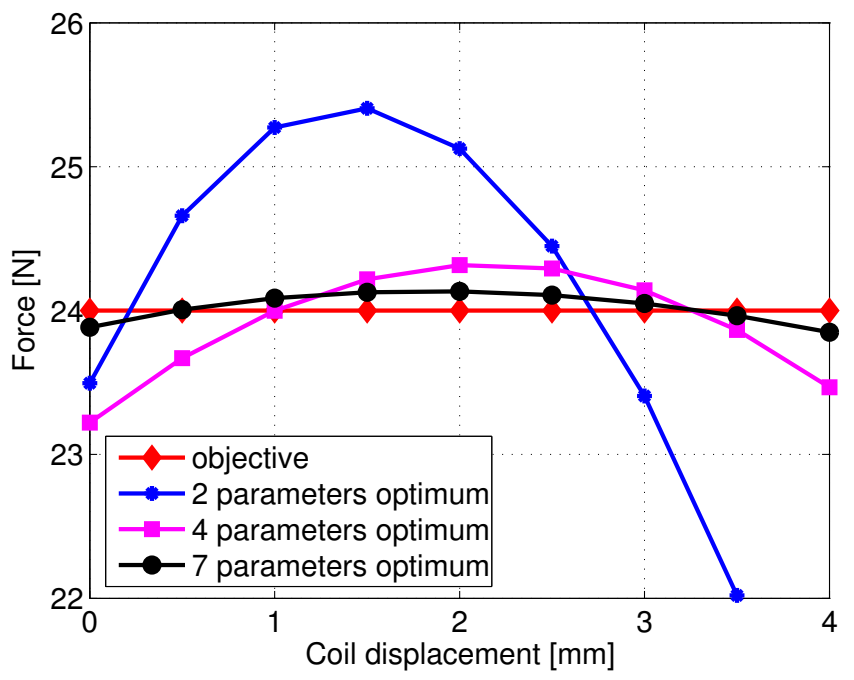

(b) Optimal force displacement curves

Figure 2: Optimal geometry and force-displacement curves for the cylindrical voice-coil actuator. 
first author is supported by the Dutch Ministry of Economic Affairs within the project IOP-EMVT 04302.

\section{References}

[1] J. W. Bandler, Q. S. Cheng, S. A. Dakroury, A. S. Mohamed, M. H. Bakr, K. Madsen, and J. Søndergaard. Space Mapping: The State of the Art. IEEE Trans. on Microwave Theory and Techniques, 52(1):337-361, 2004.

[2] G. Box and N. Draper. Empirical model-building and response surfaces. Wiley, New York, 1987.

[3] A. Canova, G. Gruosso, and M. Repetto. Magnetic design optimization and objective function approximation. IEEE. Trans. on Magn., 39(5):2154$2162,2003$.

[4] M. Dorica and D. Giannacopoulos. Response surface space mapping for electromagnetic optimization. IEEE. Trans. on Magn., 4(42):1123-1126, April 2006.

[5] D. Echeverría and P. W. Hemker. Space mapping and defect correction. Comp. Methods in Appl. Math., 5(2):107-136, 2005.

[6] D. Echeverría, D. Lahaye, L. Encica, E. A. Lomonova, P. W. Hemker, and A. J. A. Vandeput. Manifold mapping applied to linear actuator design. IEEE. Trans. on Magn., 42(4):1183-1186, 2006.

[7] D. Echeverría, D. Lahaye, L. Encica, E. A. Lomonova, P. W. Hemker, and A. J. A. Vandeput. Manifold mapping applied to linear actuator design. Technical Report E0606, CWI, 2006.

[8] P. W. Hemker and D. Echeverría. A trust-region strategy for manifold mapping optimization. Technical Report E0618, CWI, 2006.

[9] S. L. Ho, S. L. Yang, G. Z. Ni, and H. C. Wong. A response surface methodology based on improved compactly supported radial basis function and its application to rapid optimizations of electromagnetic devices. IEEE. Trans. on Magn., 41(6):2111-2117, June 2005.

[10] A. Manella, M. Nervi, and M. Repetto. Response surface method in magnetic optimization. Int. J. Appl. Electromagn. Mater., 4:99-106, 1993.

[11] R. Storn and K. Price. Differential evolution - a simple and efficient heuristic for global optimization over continuous spaces. Journal of Global Optimization, 11:341-359, 1997.

[12] N. Takahashi. Optimization of die press model. In Proc. TEAM workshop 6th Round, Okayama, Japan, March 20-21, 1996.

[13] N. Takahashi, K. Ebihara, K. Yoshida, T. Nakata, K. Ohashi, and K. Miyata. Investigation of simulated annealing method and its application to optimal design of die mold for orientation of magnetic powder. IEEE Trans. on Magn., 32(3):1210-1213, May 1996. 